\title{
Enabling Real-Time Video Services Over Ad-Hoc Networks Opens the Gates for E-learning in Areas Lacking Infrastructure
}

\author{
doi:10.3991/ijim.v3i4.911 \\ Johannes Karlsson, Adi Anani and Haibo Li \\ Umeå University, Umeå, Sweden
}

\begin{abstract}
In this paper we suggest a promising solution to come over the problems of delivering e-learning to areas with lack or deficiencies in infrastructure for Internet and mobile communication. We present a simple, reasonably priced and efficient communication platform for providing e-learning. This platform is based on wireless ad-hoc networks. We also present a preemptive routing protocol suitable for real-time video communication over wireless adhoc networks. Our results show that this routing protocol can significantly improve the quality of the received video. This makes our suggested system not only good to overcome the infrastructure barrier but even capable of delivering a high quality e-learning material.
\end{abstract}

Index Terms-E-learning, Education, Ad-hoc networks, Wireless LAN, Video coding.

\section{INTRODUCTION}

Education is a basic human right for people no matter how old they are and irrespective of their race or religion [1]. Although a lot has been made and plenty is said about equality, justice and human rights the authors believe that still there are a lot of divides and barriers in our world to overcome. The world was and still divided into Acategory with access to a wide spectrum of general and advanced education and B-category with no or perhaps a very limited access. The earlier introduction of traditional distance learning partially helped some of those of the Bcategory to get access to education. But the gap was apparent. Distance Learning, nowadays well known as eLearning is run to meet important national and international needs based upon economical, political, geographical and demographical conditions. It's the conditions of the local and global societies that motivate utilization of e-learning, not only the strained economical conditions of the individual student [2]. The transition of traditional distance learning into e-learning introduced new conditions and digital educational platforms that caused persistence in the gap in education. These platforms imply that the user has access to a terminal for communications and interaction in addition to infrastructure. A major and highly topical divide is the digital divide where some people have access and benefit from the digital technology and some don't. According to statistics from the United Nations [3] the digital divide is still wide. In developed countries, 58 per cent of the population were using the Internet in 2006, compared to 11 per cent in developing countries and only one per cent in the least developed countries. The digital divide arose as a result of the vast and fast development in information systems, digital media and communications technologies. However, we cannot put all the blame on the progress of technology; social, political, economical, environmental, geographical and demographical factors play a prominent role in this aspect. These and other global factors lead to divides among nations and some times among the same nation. The digital divide may be seen as a difference between generations where the younger generations born in the digital world are considered as natives while the older generations are considered as immigrants. Still, the most important feature, but not the only one, which characterizes the digital divide is the issue of infrastructure or more correctly the absence of it. How to deal with this issue in education is the main purpose of this paper.

How can we make education available to every one who has the right to it? Traditional learning methods in education imply physical accessibility. Educational institutions must be within safe physical reach for all involved in teaching and learning activities. As this is not always the case due to long distance as the case in the northern part of Sweden or to some specific security measures as in the Palestinian territories virtual accessibility and virtual reach become inevitable. Elearning turns to be the alternative. E-learning is the delivery of a learning, training or education program by electronic means. It involves the use of a computer or electronic device such as a mobile phone or an iPod in some way to provide training, educational or learning material. E-learning also means the use of electronic applications and processes to learn. This includes among others web-based learning, virtual classrooms and digital collaboration. Content is delivered via the Internet, intranet/extranet, audio or video tape, satellite TV, etc. Elearning nowadays is very demanding; it should maintain high social status and keep a high quality standard to meet the requirements of accreditation.

\section{A. Importance of Real-Time Video in E-Learning}

One should not underestimate the expectation of the students who for every day that goes acquire better course content and clearer presentation means. Such means are PowerPoint presentations, books, papers and video recordings. These can be well managed even with the availability of networks with limited bandwidth. However, the most demanding part is social interaction and private chat among students and even more when lecturing in real time. Real-time video is getting more and more the most 
profound way to convey course information and contents fulfilling student satisfaction. We at Umeå University together with another European university and three Mediterranean universities in the ICT-LEAP consortium gave a successful live lecture-course to students at these universities. Lectures were given by experts in their fields from the named universities based on their specialization. It was necessary to have real video during these lectures to guarantee interaction between students and lecturers. The picture below shows a lecture in action. The main problem, however, remains. How can we achieve a good quality video over wireless networks?

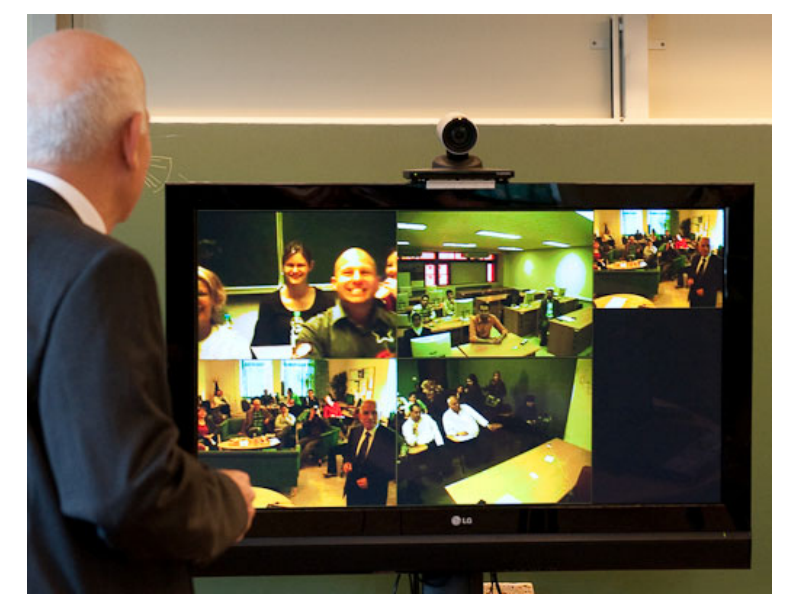

Figure 1. The opening session of the biomedia course given within the ICT-LEAP project.

Traditionally, interactive communications facilitating the use of Internet either through desk computers, laptops or mobile units as PDAs and mobile phones needs a well established communications infrastructure. M-learning is a special E-learning supporting mobility. M-learning takes wireless communication instead of wire communication in E-learning to build the network platform [4].

Infrastructure, however, is not available everywhere and thus not everyone is reached. This means that the alternative to traditional learning, e.g. e-learning or Mlearning fails to accomplish the intended goal. Populations living in areas with no infrastructure for Internet and mobile phones have no chance to learn, i.e. no chance to build a knowledge-based society. Education may not reach all; the world is going to Web2.0 and some are still at Web Zero!

It is thus apparent that to have just education through elearning two main issues need to be met simultaneously. One is the communication terminal and the other is the communication network that guarantees the real-time video necessary in live lecturing and social interaction as mentioned above. It is of no use to give a laptop, a mobile or smart telephone to a person in an area where no communication coverage exists. On the other hand it is meaningless to furnish a communication network with excellent communication coverage if people there cannot afford having a laptop or any of the known communication terminals. In this aspect there are a lot of ongoing efforts with noteworthy progress like the "One Laptop Per Child project", OLPC, and hopefully this issue will be solved when every child in the developing countries can have one. However, the other issue of network communication in spite of the numerous research projects still needs more efforts to be satisfactory solved furnishing access to every user.

In this paper we present a possible solution to come over the problem of infrastructure and student satisfaction described above. The proposed solution presents an alternative to the availability of infrastructure. It is simple, cheap and effective. It can be used alone and independent of infrastructure or in coordination with it. The suggested solution is based on wireless ad-hoc networks. It is a tailor made wireless solution that can work well together or as a complimentary to an existing or a nearby base station.

The laptops provided by the OLPC project will have the possibility to run ad-hoc networks based on the IEEE 802.11s standard [5]. However, the main purpose of that ad-hoc network is for applications having very low Quality of Service (QoS) requirements, for example email and web browsing. We suggest a new routing protocol better suited for real-time communication, for example real-time video conferencing. This will enable the possibility to provide high quality e-learning material to the users. We also suggest adding solar powered communication nodes in the network to be able to guarantee network coverage at times when the number of clients are few.

\section{B. Scenario}

Infrastructure may cover a lot of places but still there are places not covered because it is remote from the main cities and the population density is very low. People in such remote areas will not have equal opportunity even in a knowledge-based society. On the other hand there are societies where high density populations due to one reason or another do not have access to infrastructure or it is too expensive to use it. The solution of wireless ad-hoc networks can be applied to both scenarios of remote low density population and high density population. The remainder of this paper is organized as follows. In the next chapter we discuss the related research. Chapter III describes the ad-hoc network hardware platform we use to provide e-learning. In chapter IV we describe a routing protocol we have developed suitable for real-time video communication over wireless ad-hoc networks. In chapter $\mathrm{V}$ we present our experimental results. Finally chapter VI concludes our paper.

\section{RELATED RESEARCH}

There are several papers dealing with video transport over wireless ad-hoc networks where the video is split into two or more descriptions and sent over multiple paths, see for instance [6], [7], [8], [9]. This approach is most effective when there are multiple disjoint paths available between the sender and the receiver. We believe that an approach using a preemptive single-path ad-hoc routing protocol will give good real-time video performance, and this approach also works well for situations where only one path is available. A similar type of preemptive routing approach for real-time video has been implemented and demonstrated for a small scale real ad-hoc network by previous authors [10]. In our work we implement a preemptive ad-hoc routing protocol in a network simulator and shows how it performs for different network scenarios and different types of video streams. We also compare the video performance using our routing protocol to some of the most commonly used routing protocols. 


\section{ENABLING REAL-Time VideO SERVICES OVER AD-Hoc NeTWORKS \\ OPENS THE GATES FOR E-LEARNING IN AREAS LACKING INFRASTRUCTURE}

\section{PLATFORM}

In this chapter we present our platform for providing a practical and reasonable priced communication network. This platform is based on wireless ad-hoc networks and can be used to provide high quality e-learning. The platform consists of a set of nodes that are connected by wireless links. In this network the nodes are free to move and the topology of the network may therefore change. The nodes can forward data for each other in multiple hops and the path between two nodes is set up dynamically depending on the connectivity between the nodes and without the support of any central coordinator. The coverage of this network is increased when more users are added to the network. This type of network is very robust since nodes can be added or removed from the network dynamically and the routing will adjust rapidly to the new conditions. Even thought the capacity for end to end communication is decreased when data is forwarded in multiple hops [11], the capacity provided by an ad-hoc network is high enough to enable real-time video communication.

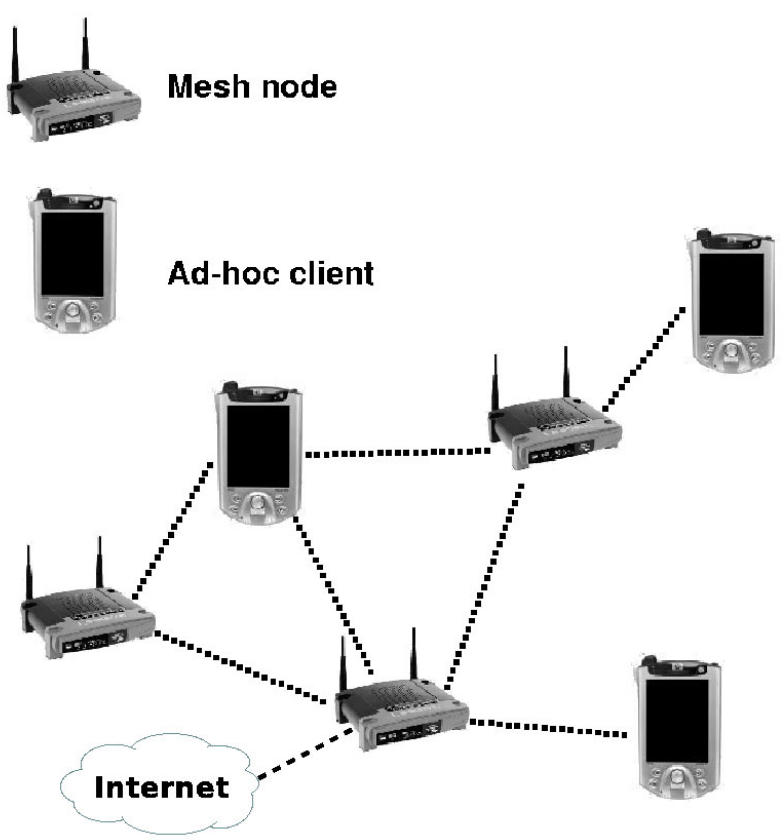

Figure 2. An overview of a network consisting of mesh nodes and mobile ad-hoc clients.

Our network is built up using two different types of nodes, see Fig. 2. One type of nodes is the ad-hoc clients in the network. These nodes can be for example a laptop equipped with an IEEE 802.11 wireless interface. This type of nodes can relay traffic for other nodes, but also be sink or source for traffic. They are both routers and hosts. These nodes are mobile and can also be switched on and off randomly thus creating fast changes in the network topology. The other type of nodes is mesh nodes that will only relay traffic for other nodes. They are needed to guarantee connectivity even at times when the number of mobile clients is few. These nodes can be located at rooftops or other positions providing a high level of coverage.

\section{A. Mesh nodes}

The requirements for the mesh nodes are a CPU capable of running the routing protocol, a wireless communication interface, possibilities to harvest energy from the environment, and a battery to compensate for the variation in energy collected from the environment. The node should be able to operate even if there is no available connection to a power line.

The mesh nodes used in this testbed are built using a Linksys WRT54GL router, a solar panel and a battery, see Fig. 3 for a picture of a deployed mesh node. All components are high volume consumer products to keep the cost for a node as low as possible.

The Linksys WRT54GL router has a $200 \mathrm{MHz}$ Broadcom 5352 CPU, 4MB Flash, 16MB RAM and an IEEE $802.11 \mathrm{~b} / \mathrm{g}$ wireless chipset. It is capable of running a Linux distribution enabling the possibility to add customized ad-hoc routing protocols. It uses a switched power regulator and at $12 \mathrm{v}$ the measured current was $240 \mathrm{~mA}$.

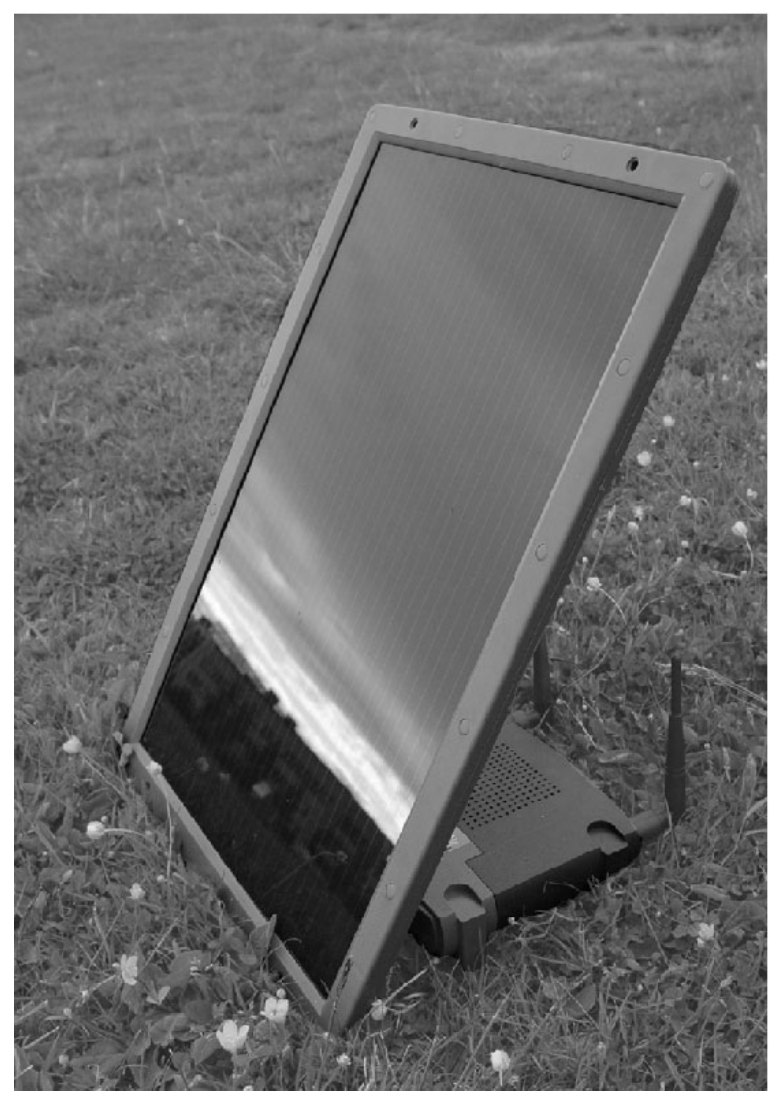

Figure 3. A picture of a deployed mesh node.

\section{B. Ad-hoc clients}

For the mobile ad-hoc clients node several suitable products are about to enter the market. The "One Laptop Per Child project", OLPC, is aiming to deliver a laptop suitable for education at the cost of 100 USD. These laptops have a 7.5“ TFT display, a VGA camera, and a wireless network card capable of creating an ad-hoc network even when the CPU is off. The Classmate PC from Intel is also developed for the same purpose. These laptops are not widely available yet and in this testbed standard laptop computers are used instead. 


\section{ENABLing REAL-Time VideO SERVICES OVER AD-Hoc NETWORKS \\ OPENS THE GATES FOR E-LEARNING IN AREAS LACKING INFRASTRUCTURE}

\section{ROUTING}

Sending real-time video over wireless ad-hoc networks is a challenging problem. Video is very sensitive for packet loss and wireless ad-hoc networks are error prone due to node mobility and weak links. The most popular routing protocols today for ad-hoc networks, for example Dynamic Source Routing (DSR) [12] and Ad-hoc On Demand Distance Vector (AODV) [13] are reactive routing protocols that are focused on scalability for very large networks and to reduce the routing load [14]. The major problem when sending real-time video over reactive routing protocols is however the long interruption in packet delivery when a route breaks and a new route has to be established. This is a drawback of the reactive design. To achieve high robustness for real-time video over ad-hoc networks issues like the delay and packet delivery ratio are very important.

We have developed a preemptive routing protocol by adding some extensions to standard DSR. We call this version of DSR for real-time DSR (RT-DSR). There are basically two problems with the standard DSR that this new routing protocol solves. The first problem is that DSR will not search for new routes until a route breaks. During the time it takes to discover new routes no data can be delivered. This is an unacceptable delay for the real-time video. The other problem is the gray-zone problem [15]. The standard DSR routing protocol often discovers routes that contains very weak links. The route packets are broadcasted and they are relatively small compared to the data packets.

We start with the most basic form of DSR. The following optimizations were removed from DSR:

- Automatic route shortening

- Caching overheard routing information

- Replying to route requests using cached routes

- Overhearing of packets

- Adding routes to intermediate nodes

- Gratuitous Route Replies

- All nodes process all of the Route Error messages they receive

If the event of routes that are about to break can be discovered it is possible to establish and start to use new routes before the old routes break. By simply monitoring the signal strength for each data packet we can detect if a route is about to break. To avoid using very weak links when establishing new routes the Route Request (RREQ) packets are filtered based on received signal strength.

The following two extensions were added to DSR:

- Send a special "weak RERR" packet when a data packet having low signal strength is routed

- Signal strength threshold for RREQ packets

The modifications to the data structures, packet formats and algorithms are described in the following sections.

\section{A. Data structures}

When the source node receives information about a weak link on a route this route should still be used until new routes have been discovered. The old routes must however be removed after the new routes have been discovered. If this is not done, the old routes will be continued to be used until a packet is dropped on the route. We therefore add a 1-bit flag, weakSignal, to the route cache. This flag is used to mark the routes to be deleted after new routes have been discovered.

\section{B. Packet formats}

A control packet is used to inform the source of the route when a weak link on the route is detected. If we use a regular RERR packet all intermediate nodes and the source node receiving the RERR packet will remove all routes in its route cache containing the weak link. This should not happen until new routes have been discovered. Instead of creating a complete new control packet a weakSignal flag is added to the RERR packet. The RERR packet has an 8-bit Error Type field and a 4-bit Reserved field that can be used for this.

\section{Route maintenance}

A RERR packet with the weakSignal flag set is initiated every time a node receives a data packet having a signal strength below $-80 \mathrm{dBm}$. When the source or intermediate node receives this RERR packet they will not delete any routes in the route cache. When the source receives this RERR packet it will first set the weakSignal flag for all routes in the route cache for this destination. The source node will then initiate a route discovery by sending a Route Request (RREQ) packet. When the node receives a Route Reply (RREP) it will remove all routes in its route cache having the weakSignal flag set for the given destination.

\section{Route discovery}

When a route discovery is performed it is desired to avoid discovering routes containing weak links. Because the control packets are small and broadcasted they are less error prone than the data packets. If all RREQ packets are forwarded it is likely that many of the discovered routes will contain links having a very high packet loss. This is referred to the gray-zone problem in wireless ad-hoc networks. We therefore discard RREQ packets received with a signal strength below $-80 \mathrm{dBm}$.

\section{SimUlations}

The GloMoSim 2.03 [16] network simulator is used in our simulations. This is an event driven network simulator developed at the UCLA parallel computing laboratory. We have one version for the original DSR routing protocol and one version for the modified RT-DSR routing protocol. We also compared our results with the AODV [13] routing protocol.

For all the simulations we used the following settings:

PROPAGATION-LIMIT -111.0

PROPAGATION-PATHLOSS TWO-RAY

NOISE-FIGURE 10.0

TEMPARATURE 290.0

RADIO-TYPE RADIO-ACCNOISE

RADIO-FREQUENCY 2.4e9

RADIO-BANDWIDTH 11000000

RADIO-RX-TYPE SNR-BOUNDED

RADIO-RX-SNR-THRESHOLD 10.0

RADIO-TX-POWER 15.0

RADIO-ANTENNA-GAIN 0.0

RADIO-RX-SENSITIVITY -91.0

RADIO-RX-THRESHOLD -81.0

MAC-PROTOCOL 802.11 
The two-ray path loss model was used (3). This is the maximum of the plane earth (1) and the free space path loss (2),

$$
\begin{gathered}
P=\frac{d^{2}}{t \times r}, \\
S=\frac{4 \times \pi \times d}{\lambda}, \\
T=\max (P, S),
\end{gathered}
$$

Where $d$ is the distance between the sender and receiver, $t$ is the tx antenna height, $r$ is the rx antenna height and $\lambda$ is the signal wavelength. In GloMoSim both the rx and tx antenna height is 1.5 meters and the wavelength in this simulation was set to 0.125 meters. When the distance is 356 meters the received signal strength will be $-80.01 \mathrm{dBm}$ and the link will be detected as a weak link. When the distance is 377 meters the received signal strength will be $-81.01 \mathrm{dBm}$, this is below the radio rx threshold and the packet will be dropped.

In our simulations we used the theora video codec and the ogg container format. We used two different videos in our simulations. One was the commonly used foreman video sequence in qcif resolution (176x144 pixels). The original video clip was 400 frames at 30 frames per second and we looped this sequence 45 times to create a 600 second and 18000 frames long video clip. The other video we used was the first 600 seconds from the opening session of the biomedia course given within the ICTLEAP consortium. This course is given for five universities over video conferencing networks. We used the recorded video from a video conference session and encoded the video from one of the parties at qcif resolution. For both of the video clips the video was encoded using a target bitrate $100 \mathrm{kbit} / \mathrm{s}$, keyframe interval 120 and 30 frames per second. The actually encode bitrate, including the ogg headers, was $157.1 \mathrm{kbit} / \mathrm{s}$ for the foreman sequence and $101.4 \mathrm{kbit} / \mathrm{s}$ for the biomedia course sequence. First a trace file was created. This file contained the size of each packet and the time for transmission. This file was used in the GloMoSim simulations and the lost packets were recorded. This information was then used when the video was decoded. If a packet was lost or had a delay of more than $50 \mathrm{~ms}$ the packet was dropped at decoding. The error concealment method used at the decoder was to copy the previous successfully decoded frame if a frame was lost. To compare the new routing protocol to AODV and standard DSR we run three different simulation scenarios. In each simulation we recorded the number of lost packets, the average PSNR, the number of frames having a PSNR below $30 \mathrm{~dB}$ and the total number of routing packets transmitted. An error free transmission had an average PSNR of $34.87 \mathrm{~dB}$ for the foreman video sequence and $40.79 \mathrm{~dB}$ for the biomedia course video sequence. The foreman video sequence had much more motion, including camera motion, compared to the video sequence from the biomedia course and since the video was coded using constant bitrate the encoded quality of the forman video was lower.

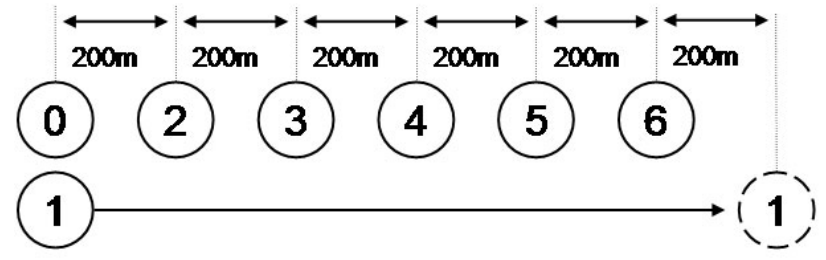

Figure 4. The roaming node scenario.

\section{A. Roaming node}

In this scenario we forced a number of route breaks at the last hop. We used a total of seven nodes. The nodes were placed on a row having a distance of 200 meters between them, see Fig. 4 . The source and the destination node had the same initial position. The destination node was then moving along the row of nodes at a speed of 2 $\mathrm{m} / \mathrm{s}$ until it was located 200 meters to the right of node six. First there was a one-hop link between the source node and the destination node. When the destination node was located between node two and node three the one-hop route was lost and a two-hop route had to be established. Similarly the two-hop, three-hop, four-hop and five-hop route was lost. Finally before the destination node reached its final destination 200 meters to the right of node six the five-hop route was lost and a six-hop route had to be established. There were a total of five route breaks in this simulation.

The simulation time was 600 seconds and we run the simulation ten times for each video sequence with different initial seed.

TABLE I.

ROUTE PERFORMANCE IN THE ROAMING NODE SCENARIO AND THE FOREMAN VIDEO CLIP

\begin{tabular}{|c|l|l|l|}
\hline & \multicolumn{1}{|c|}{ RT-DSR } & \multicolumn{1}{|c|}{ DSR } & \multicolumn{1}{|c|}{ AODV } \\
\hline Lost packets & $0.00 \%$ & $11.05 \%$ & $0.30 \%$ \\
\hline PSNR (dB) & 34.87 & 25.68 & 34.10 \\
\hline PSNR $<30 d B$ & $0.00 \%$ & $14.07 \%$ & $1.20 \%$ \\
\hline Control packets & 149 & 5004 & 61 \\
\hline
\end{tabular}

TABLE II.

ROUTE PERFORMANCE IN THE ROAMING NODE SCENARIO AND THE BIOMEDIA COURSE VIDEO CLIP

\begin{tabular}{|c|l|l|l|}
\hline & \multicolumn{1}{|c|}{ RT-DSR } & \multicolumn{1}{|c|}{ DSR } & \multicolumn{1}{|c|}{ AODV } \\
\hline Lost packets & $0.00 \%$ & $11.52 \%$ & $0.29 \%$ \\
\hline PSNR (dB) & 40.79 & 30.89 & 40.26 \\
\hline PSNR $<$ 30dB & $0.81 \%$ & $12.97 \%$ & $0.92 \%$ \\
\hline Control packets & 148 & 5224 & 61 \\
\hline
\end{tabular}

In table I and table II we can see that RT-DSR had much lower packet loss compared to standard DSR for both video sequences. Most of the packet losses when DSR was used occurred at the last route breaks. The RTDSR routing also outperformed the AODV routing protocol for both video sequences, the difference was however much smaller compared to DSR.

\section{B. Intermediate break}

In this scenario we forced link breaks at intermediate nodes. Five nodes were located on a row having a distance 
of 200 meters between each node, see Fig. 5. This created a four-hop path between node zero and node one. When the simulation started node five was located in the middle of the path. It then moved at the speed of $4 \mathrm{~m} / \mathrm{s}$ away from the path until it was located 400 meters from its original location. At the same time node four was moving to node fives original location. After this both node four and node five moved back to its starting locations at a speed of 4 $\mathrm{m} / \mathrm{s}$. This was repeated three times and a total of six route breaks was generated.

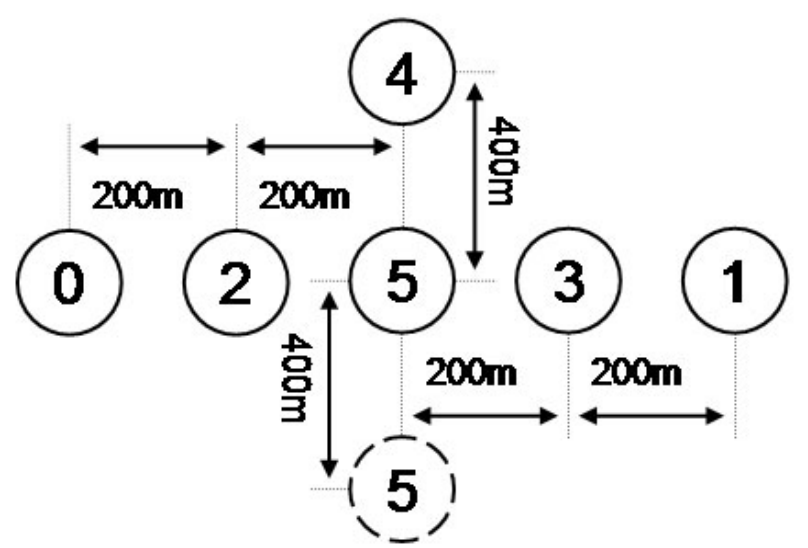

Figure 5. The intermediate node break scenario.

The total simulated time was 600 seconds and we repeated the simulation ten times for each video sequence with different initial seed. In this simulation the route was first established by sending a data packet before the video transmission was started.

TABLE III.

ROUTE PERFORMANCE IN INTERMEDIATE BREAK SCENARIO AND THE FOREMAN VIDEO CLIP

\begin{tabular}{|c|l|l|l|}
\hline & \multicolumn{1}{|c|}{ RT-DSR } & \multicolumn{1}{|c|}{ DSR } & \multicolumn{1}{|c|}{ AODV } \\
\hline Lost packets & $0.00 \%$ & $0.01 \%$ & $0.00 \%$ \\
\hline PSNR (dB) & 34.87 & 34.81 & 34.87 \\
\hline PSNR $<$ 30dB & $0.00 \%$ & $0.06 \%$ & $0.00 \%$ \\
\hline Control packets & 194.7 & 52.6 & 60 \\
\hline
\end{tabular}

TABLE IV.

ROUTE PERFORMANCE IN INTERMEDIATE BREAK SCENARIO AND THE BIOMEDIA COURSE VIDEO CLIP

\begin{tabular}{|c|l|l|l|}
\hline & \multicolumn{1}{|c|}{ RT-DSR } & \multicolumn{1}{|c|}{ DSR } & \multicolumn{1}{|c|}{ AODV } \\
\hline Lost packets & $0.00 \%$ & $0.00 \%$ & $0.00 \%$ \\
\hline PSNR (dB) & 40.79 & 40.79 & 40.79 \\
\hline PSNR $<$ 30dB & $0.81 \%$ & $0.81 \%$ & $0.81 \%$ \\
\hline Control packets & 185 & 52.7 & 60 \\
\hline
\end{tabular}

In table III and table IV we can see that all routing protocols, especially DSR, performed much better compared to the roaming node scenario. As expected the RT-DSR has the highest number of routing control packets.

\section{Random waypoint}

In this simulation we let a number of nodes move randomly within an area. We used a very high node density to make the network connected with high probability. This is because we did not want to have dropped packets because there were no routes available between the source and the destination. We used 60 nodes distributed within an area of size 1000x1000 meters. The nodes were initially distributed using uniform node placement. The mobility model used was random waypoint. Both the max and min speed were set to $4 \mathrm{~m} / \mathrm{s}$. The waypoint pause time was set to 1 second. The simulation was run for 600 seconds and each simulation was repeated ten times for each video sequence with different initial seed.

In table V and table VI we can see that the RT-DSR had less dropped packets compared to both DSR and AODV. Even tough we used high node density it is likely that some of the packets were lost because no route existed between the source and the destination. The RT-DSR routing protocol had much higher routing overhead compared to both DSR and AODV in this simulation too.

TABLE V.

ROUTE PERFORMANCE IN THE RANDOM WAYPOINT SCENARIO AND THE FOREMAN VIDEO CLIP

\begin{tabular}{|c|l|l|l|}
\hline & \multicolumn{1}{|c|}{ RT-DSR } & \multicolumn{1}{c|}{ DSR } & \multicolumn{1}{c|}{ AODV } \\
\hline Lost packets & $0.04 \%$ & $3.25 \%$ & $0.15 \%$ \\
\hline PSNR (dB) & 34.77 & 31.05 & 34.37 \\
\hline PSNR $<$ 30dB & $0.26 \%$ & $4.72 \%$ & $1.03 \%$ \\
\hline Control packets & 1483 & 751 & 382 \\
\hline
\end{tabular}

TABLE VI.

ROUTE PERFORMANCE IN THE RANDOM WAYPOINT SCENARIO AND THE BIOMEDIA COURSE VIDEO CLIP

\begin{tabular}{|c|l|l|l|}
\hline & \multicolumn{1}{|c|}{ RT-DSR } & \multicolumn{1}{c|}{ DSR } & \multicolumn{1}{|c|}{ AODV } \\
\hline Lost packets & $0.03 \%$ & $2.71 \%$ & $0.17 \%$ \\
\hline PSNR (dB) & 40.71 & 38.10 & 40.15 \\
\hline PSNR $<$ 30dB & $0.82 \%$ & $2.87 \%$ & $0.97 \%$ \\
\hline Control packets & 1778 & 838 & 422 \\
\hline
\end{tabular}

\section{CONCLUSION}

In this paper we have presented a possible solution for providing high quality e-learning to people living in areas with no infrastructure for Internet and mobile communication or where the cost to use the existing infrastructure is too high. This solution is based on wireless ad-hoc networks. The hardware platform presented is built using low cost high volume consumer products.

A preemptive ad-hoc routing protocol is also presented in this paper. This routing protocol was developed to increase the real-time video performance in ad-hoc networks. Our result shows that the packet loss was decreased when our routing protocol was used. In realtime video a lost packet will cause error propagation into following frames and the number of frames having a low quality was therefore significantly much higher than the number of lost frames.

Applying this system in practice would probably make it possible to reach every body interested in using his or her right in education independent of the place of living or surrounding environment.

\section{REFERENCES}

[1] "The universal declaration of human rights, general assembly resolution 217 a (iii) of 10 december 1948,"

[2] Adi Anani, "A Distance Learning Program in Action", Proceedings of the 29th IEEE Frontiers in Education Conference, November 10 - 13, 1999 San Juan, Puerto Rico 


\section{ENABLing REAL-Time VideO SERVICES OVER AD-Hoc NETWORKS \\ OPENS THE GATES FOR E-LEARNING IN AREAS LACKING INFRASTRUCTURE}

[3] "The Millennium Development Goals Report 2008", United Nations.

[4] Adi Anani et al, "M-learning in review: Technology, standard and evaluation", Journal of Communication and Computer, vol 5, no.11, November 2008.

[5] Michael Bahr, "Proposed routing for ieee 802.11s wlan mesh networks," in WICON '06: Proceedings of the 2nd annual international workshop on Wireless internet, New York, NY, USA, 2006, p. 5, ACM Press.

[6] Shiwen Mao et al, "On Routing for Multiple Description Video Over Wireless Ad Hoc Networks", IEEE Transaction on Multimedia, Vol. 8, No. 5, October 2006.

[7] Wei Wei and Avideh Zakhor, "Multipath Unicast and Multicast Video Communication over Wireless Ad Hoc Networks" in International Conference on Broadband Networks (Broadnets) 2004, San Jose, CA, October 2004, pp. 496-505. (invited paper)

[8] Song Xiao, Chengke $\mathrm{Wu}$, and Fang Zhang, "A new multiple description layered coding method over ad-hoc network", in Proceedings of the 2004 International Conference on Image Processing (ICIP'04), pp. 3229- 3232.

[9] P. Seeling and M. Reisslein, "Video Coding with Multiple Descriptors and Spatial Scalability for Device Diversity in Wireless Multi-hop Networks", In Proceedings of the IEEE Consumer Communications and Networking Conference (CCNC), pages 278-283, Las Vegas, NV, January 2005.

[10] Yih-Chun Hu and David B. Johnson, "Design and Demonstration of Live Audio and Video over Multihop Wireless Ad Hoc Networks", Proceedings of the MILCOM 2002 IEEE Military Communications Conference, IEEE, Anaheim, CA, October 2002.

[11] Jinyang Li, Charles Blake, Douglas S. J. De Couto, Hu Imm Lee, and Robert Morris, "Capacity of ad hoc wireless networks," in
Proceedings of the 7th ACM International Conference on Mobile Computing and Networking, Rome, Italy, July 2001, pp. 61-69.

[12] David B Johnson and David A Maltz, "Dynamic source routing in ad hoc wireless networks," in Mobile Computing, vol. 353. Kluwer Academic Publishers, 1996.

[13] C. Perkins, E. Belding-Royer, and S. Das, "Ad hoc on-demand distance vector (aodv) routing," RFC 3561, July 2003.

[14] Chareles E. Perkins, Elizabeth M. Royer, Samir R. Das, and Mahesh K. Marina, "Performance comparation of two ondemand routing protocols for ad hoc networks," IEEE Personal Communications Special Issue on Advances in Mobile Ad Hoc Networking, vol. 8, no. 1, February 2001.

[15] Henrik Lundgren, Erik Nordstrm, and Christian Tschudin, "Coping with communication gray zones in ieee $802.11 \mathrm{~b}$ based ad hoc networks," in 5th ACM international workshop on Wireless mobile multimedia (WoWMoM 2002. 2002, pp. 49-55, ACM Press.

[16] Xiang Zeng, Rajive Bagrodia, and Mario Gerla, "Glomosim: A library for parallel simulation of large-scale wireless networks," in Workshop on Parallel and Distributed Simulation, 1998, pp. 154161.

\section{AUTHORS}

J. Karlsson, A. Anani and H. Li are with the Digital Media Laboratory (DML), Department of Applied Physics and Electronics, Umeå University, SE-90187, Umeå Sweden e-mail: \{johannes.karlsson, adi.anani, haibo.li\}@tfe.umu.se

Submitted 30 April 2009. Published as resubmitted by the authors on 9 October 2009. 